\title{
A NOTE ON THE INVERSE FUNCTION THEOREM OF NASH AND MOSER
}

\author{
MIKAEL LINDSTROİ \\ Department of Mathematics \\ Ábo Akademi \\ SF-20500 Åbo, Finland \\ (Received September 9, 1985)
}

ABSTRACT. The Nash-Moser inverse function theorem is proved for different kind of differentiabilities.

KEY WORDS AND PHRASES. Continuous convergence, coreflective subcategory, graded space, smoothing operators, tame map, $C_{\alpha}^{m}$-differentiability. 1980 MATHEMATICS SUBJECT CLASSIFICATION CODE. $58 C 15$.

\section{INTRODUCTION}

The purpose of this note is to formulate the inverse function theorem of Nash and Moser for different differentiabilities using a categorical approach. The proof is based on the inverse function theorem of Nash and Moser in the version of Hamilton [1] formulated in the category of graded Fréchet spaces which admit smoothing operators and $\mathrm{C}_{\mathrm{c}}^{\infty}$-differentiable [2] tame maps. Our proof is using the same technique as Schmid [3] uses when he proves this theorem for a stronger notion of differentiability, called the $\Gamma$-differentiability, than the $C_{c}^{\infty}$-differentiability. From our formulation it is possible to derive the inverse function theorem of Nash and Moser for natural differentiabilities stronger than the $\mathrm{C}_{\mathrm{C}}^{\infty}$-differentiability.

2. THE INVERSE FUNCTION THEOREM OF NASH AND MOSER.

Let LC denote the category of locally convex limit vector spaces [2] and continuous linear mappings. Further let $K_{\alpha}$ denote a coreflective subcategory of LC which is closed under finite products and the coreflector $?^{\alpha}: \mathrm{LC} \rightarrow K_{\alpha}$ is the identity on morphisms and such that the identity mapping $\left(C_{C}(X, F)\right)^{\alpha}=C_{\alpha}(X, F) \rightarrow C_{c}(X, F)$ is

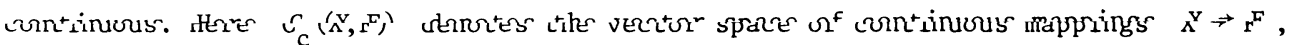
endowed with continuous convergence [2], and $X$ is a limit space and $F \in$ obj(LC).

For any pair $E, F \in$ obj $(L C)$ we let $L_{c}^{k}(E, F)$ be the space of all continuous $k$-linear mappings from $E^{k}$ into $F$, endowed with continuous convergence. We write $\left(\mathrm{L}_{\mathrm{c}}^{\mathrm{k}}(\mathrm{E}, \mathrm{F})\right)^{\alpha}=\mathrm{L}_{\alpha}^{\mathrm{k}}(\mathrm{E}, \mathrm{F})$.

DEFINITION. Let $E$ and $F$ be locally convex spaces and let $U$ be open in $E$. 
A mapping $\mathrm{f}: \mathrm{U} \rightarrow \mathrm{F}$ is said to be differentiable of class $\mathrm{C}_{\alpha}^{\mathrm{p}}$, if there exist functions

$$
D^{k_{f}}: U \rightarrow L^{k}(E, F), \quad k=0,1, \ldots, P,
$$

such that $D^{\circ} f=f$ and for each $x \in U$, each $h \in E$ and each $k=0,1, \ldots, p-1$, we have

$$
\lim _{t \rightarrow 0} t^{-1}\left(D^{k} f(x+t h)-D^{k} f(x)\right)=D^{k+1} f(x) h
$$

and such that for each $k \in \mathbb{N}, k \leq p$, the following two conditions are satisfied:

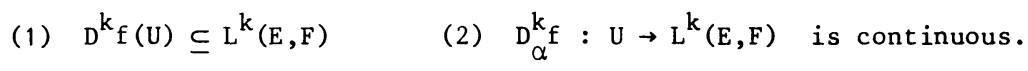

$f$ is called defferentiable of class $C_{\alpha}^{\infty}$ if it is differentiable of class $C_{\alpha}^{p}$ for every $p \in \mathbb{N}$.

By Keller [2] the chain rule is valid for $c_{\alpha}^{\infty}$, since $\alpha$ is a finer limit structure than continuous convergence. From the universal property of continuous convergence follows that for any continuous map $g: U \rightarrow L_{\alpha}^{k}(E, F)$ the associated map $\tilde{\mathrm{g}}: U \times E^{\mathrm{k}} \rightarrow \mathrm{F}$ defined by $\tilde{\mathrm{g}}\left(\mathrm{x}, \mathrm{h}_{1}, \ldots, \mathrm{h}_{\mathrm{k}}\right)=\mathrm{g}(\mathrm{x})\left(\mathrm{h}_{1}, \ldots, \mathrm{h}_{\mathrm{k}}\right), \mathrm{x} \in \mathrm{U}, \mathrm{h}_{\mathrm{i}} \in \mathrm{E}$, is continuous. As the limit structure $\alpha$ is always finer than $c$, we have that differentiability of class $c_{\alpha}^{\infty}$ implies differentiability of class $c_{c}^{\infty}$. The latter is exactly the concept of differentiability used by Hamilton [1] to prove the inverse function theorem of Nash and Moser.

We first recall some definitions that will be needed.

Let $\mathrm{E}$ be a Fréchet space. A grading on $\mathrm{E}$ is an increasing sequence of norms $\left(\|\cdot\| \begin{array}{l}1 \\ r\end{array}\right)_{r \in \mathbb{N}}$ on $E$ which defines the topology on $E$. Two gradings $\left(\|\cdot\| \begin{array}{l}1 \\ r\end{array}\right) r \in \mathbb{N}$ and $\left(\|\cdot\|_{r}^{2}\right)_{r \in \mathbb{N}}$ are equivalent if for some $s \in \mathbb{N}\|x\|_{r}^{1} \leq c\|x\|_{r+s}^{2}$ and $\|x\|_{r}^{2} \leq c\|x\|_{r+s}^{2}$, $x \in E$, with a constant $c$ which may depend on $r$. A graded space is a Fréchet space together with an equivalence class of gradings. We say that a graded space $E$ admits smoothing operators if we can find linear maps $S_{t}: E \rightarrow E, 1 \leq t<\infty$, such that for some $r\left\|s_{t}(x)\right\|_{i+k} \leq c t^{r+k}\|x\|_{i}$ and $\left\|s_{t}(x)-x\right\|_{i} \leq c t^{r-k}\|x\|_{i+k}$ for all $i, k \in \mathbb{N}, 1 \leq t<\infty, x \in E$ and some constant $c$ which may depend on $i$ and $k$. Let $E$ and $F$ be graded spaces and $U$ open in $E$. We say that a map $F: U \rightarrow F$ is tame if for every $x_{0} \in U$ we can find a neighbourhood $U_{0}$ and a number $r \in \mathbb{N}$ such that for every $n \in \mathbb{N}$ we have the growth estimate $\|f(x)\|_{n} \leq c\left(\|x\|_{n+r}+1\right)$ for a11 $x_{0} \in U$, where the constant $c$ may depend on $n$.

In the proof of the inverse function theorem of Nash and Moser we shall also need the following result (Lemma 2, [3]): The composition of two continuous tame maps is continuous and tame.

THEOREM. Let $E$ and $F$ be graded spaces which admit smoothing operators. Let $\mathrm{U}$ be open in $\mathrm{E}$ and assume that

(1) $\mathrm{f}: \mathrm{U} \rightarrow \mathrm{F}$ is differentiable of class $\mathrm{C}_{\alpha}^{\infty}$ and tame.

(2) $\widetilde{D^{k}}: U \times E^{k} \rightarrow F$ is tame for every $k \in \mathbb{N}$.

(3) For each $x \in U$ the derivative $D f(x): E \rightarrow F$ is an isomorphism.

(4) The map Vf: $U \rightarrow L_{\alpha}(F, E), V f(x)=(D f(x))^{-1}$, is continuous.

(5) $\widetilde{\mathrm{Vf}}: \mathrm{U} \times \mathrm{F} \rightarrow \mathrm{E}$ is tame. 
Then for any $x_{0} \in U$ we can $f$ ind open neighbourhoods of $x_{0}$ and $V_{0}$ of $f\left(x_{0}\right)$ such that $f$ is a bijective map from $U_{0}$ onto $\mathrm{V}_{0}$ and the inverse map $\mathrm{f}^{-1}: \mathrm{V}_{0} \rightarrow \mathrm{U}_{0}$ is differentiable of class $\mathrm{C}_{\alpha}^{\infty}$ and the maps $\overparen{\mathrm{D}^{\mathrm{f}^{-1}}}: \mathrm{V}_{0} \times \mathrm{F}^{\mathrm{k}} \rightarrow \mathrm{E}$ are tame for all $\mathrm{k} \in \mathbb{N}$. Furthermore we have the formula $D\left(f^{-1}\right)(y)=V f\left(f^{-1}(y)\right)$ for all y $\in V_{0}$.

PROOF. The maps $\overparen{\mathrm{D}_{\mathrm{f}}}: \mathrm{U} \times \mathrm{E}^{\mathrm{k}} \rightarrow \mathrm{F}$ are continuous and tame, since $\mathrm{f}$ is differentiable of class $C_{\alpha}^{\infty}$ and assumption (2). Further the assumptions (4) and (5) imply that also $\widetilde{\mathrm{Vf}}: \mathrm{U} \times \mathrm{F} \rightarrow \mathrm{E}$ is continuous and tame. Now we have that $\mathrm{f}$ is differentiable of class $C_{\alpha}^{\infty}$ and all $D^{k} f$ are tame, $D f(x): E \rightarrow F$ is an isomorphism for every $x \in U$ and the family of inverses $\widetilde{V f}: U \times F \rightarrow E$ are continuous and tame maps. Consequently the conditions of the inverse function theorem of Nash-Moser are fulfilled (theorem $1.1 .1 \mathrm{p} .171 \mathrm{in}[1])$. Then for every $x_{0} \in U$ there exist neighbourhoods $U_{0}$ of $x_{0}$ and $v_{0}$ of $f\left(x_{0}\right)$ such that $f: U_{0} \rightarrow v_{0}$ is bijective and $f^{-1}: V_{0} \rightarrow U_{0}$ is continuous and tame. Furthermore the formula $\lim _{t \rightarrow 0} t^{-1}\left(f^{-1}(y+t w)-f^{-1}(y)\right)=\operatorname{Vf}\left(f^{-1}(y)\right) w$ holds, for all $y \in V_{0}$ and $w \in F$, by the proof of theorem 1.1.1 p. 186 in [1]. By induction on $k$ we will prove the remaining part that $\mathrm{f}^{-1}: \mathrm{V}_{0} \rightarrow \mathrm{U}_{0}$ is differentiable of class $\mathrm{C}_{\alpha}^{\infty}$ and $\mathrm{D}_{\mathrm{f}}^{-1}: \mathrm{V}_{0} \times \mathrm{F}^{\mathrm{k}} \rightarrow \mathrm{E}$ is tame for every $\mathrm{k} \in \mathbb{N}$. From the formula $\mathrm{Df}^{-1}=$ $\mathrm{Vf} \circ \mathrm{f}^{-1}$ and assumption (4) follow that $\mathrm{Df}^{-1}: \mathrm{V}_{0} \rightarrow \mathrm{L}(\mathrm{F}, \mathrm{E})$ is continuous. Further we have that $\overparen{\mathrm{Df}^{-1}}: \mathrm{V}_{0} \times \mathrm{F} \rightarrow \mathrm{E}$ is tame since $\widetilde{\mathrm{Vf}}$ and $\mathrm{f}^{-1}$ are tame. Assume now it to be true for $k$. From the definition of the a-differentiability follows that the $\operatorname{map} \mathrm{f}^{-1}$ is $\mathrm{C}_{\alpha}^{\mathrm{k}+1}$ if $\mathrm{Df}^{-1}$ is differentiable of class $\mathrm{C}_{\alpha}^{\mathrm{k}}$. Since $\mathrm{Df}^{-1}=\mathrm{Vf}^{\circ} \circ \mathrm{f}^{-1}$, $\mathrm{D}^{\mathrm{k}+1} \mathrm{f}^{-1}$ is clearly tame so we only have to show that $\mathrm{Vf}$ is differentiable of class $\mathrm{C}^{\mathrm{k}}$. By induction on $\mathrm{p}$. By theorem 5.3.1, p. 102 in [1] we have that Vf is weakly differentiable and that $\overparen{D(V f)}: U_{0} \times E \times F \rightarrow E$ is continuous and the formula $[D(V f)](x)\{u, w\}=-V f(x)\left[D^{2} f(x)\{u, V f(x) w\}\right]$ holds for all $x \in U_{0}, u \in E$ and $w \in F$. Thus the derivative $\mathrm{D}(\mathrm{Vf}): \mathrm{U}_{0} \rightarrow \mathrm{L}_{\alpha}(\mathrm{E} \times \mathrm{F}, \mathrm{E})$ can be factorized according to

$$
\mathrm{U}_{0} \stackrel{\left(\mathrm{D}^{2} \mathrm{f}, \mathrm{Vf}\right)}{\longrightarrow} \mathrm{L}_{\alpha}^{2}(\mathrm{E}, \mathrm{F}) \times \mathrm{L}_{\alpha}(\mathrm{F}, \mathrm{E}) \stackrel{\mathrm{h}}{\longrightarrow} \mathrm{L}_{\alpha}(\mathrm{E} \times \mathrm{F}, \mathrm{E}) \text {, }
$$

where $h$ is defined by $h(\phi, \psi)=-\psi \circ \phi \circ\left(\operatorname{id}_{E}, \psi\right)$ for $\phi=D^{2} f(x)$ and $\psi=\operatorname{Vf}(x)$. By theorem 0.3 .5 in [2] $h$ is continuous for $\alpha=c$. Since the category $k_{\alpha}$ is closed under finite products and $?^{\alpha}$ is a coreflector it follows that $h$ is continuous. Thus it is true for $p=1$. Since $h$ is bilinear it is differentiable of class $c_{\alpha}^{\infty}$, and consequently the map $\mathrm{Vf}$ is differentiable of class $C_{\alpha}^{\infty}$ by induction. Thus the theorem is proved.

We shall now consider examples of coreflective subcategories of LC which are closed under finite products and the coreflectors $?^{\alpha}$ fulfill the assumption that the identity mapping $\mathrm{C}_{\alpha}(\mathrm{U}, \mathrm{F}) \rightarrow \mathrm{C}_{\mathrm{C}}(\mathrm{U}, \mathrm{F})$ is continuous.

EXAMPLE 1. Let $K_{\alpha}$ be the category $K_{c}=$ LC; $?^{\alpha}$ is the identity functor ${ }^{1}{ }_{L C}=?^{c}$.

EXAMPLE 2. Let $K_{\alpha}$ be the category $K_{e}$ of equable locally convex limit vector spaces [2]. The coreflector ${ }^{\mathrm{e}}: \mathrm{LC} \rightarrow K_{\mathrm{e}}$ is the identity on morphisms and on objects $E$ it is characterized as follows: a filter $F$ on $E$ converges to zero in $E^{e}$ iff $\mathbb{V} G=G \leq F$ for some filter $G$ which converges to zero in $E$. 
EXAMPLE 3. Let $K$ be the category $K_{M}$ of Marinescu spaces [2]. The corflector $?^{M}:$ LC $\rightarrow K_{M}$ is the identity on morphisms and on objects $E$ it is characterized as follows: a filter $F$ on $E$ converges to zero in $E^{M}$ iff $y G=G \leq F$ and $\cap\{\mathbb{K G}: G \in G\} \in G$ for some filter $G$ which converges to zero in $E$.

EXAMPLE 4. Let $K_{\alpha}$ be the category $K_{b}$ of bornological locally convex limit vector spaces. The coreflector $?^{b}: L C \rightarrow K_{b}$ is the identity on morphisms and on objects $E$ it is characterized as follows: a filter $F$ on $E$ converges to zero in $\mathrm{E}^{\mathrm{b}}$ iff $\mathrm{VB} \leq F$ for some bounded subset $\mathrm{B} \subseteq \mathrm{E}$, i.e. some set $\mathrm{B}$ such that $\mathrm{VB}$ converges to zero in $\mathrm{E}$.

Example 1 gives us the inverse function theorem of Nash and Moser by Hamilton [1]. From example 3 we derive the inverse function theorem of Nash and Moser for the differentiability of class $C_{M}^{\infty}\left(C_{\Delta}^{\infty}\right.$ in Keller [2]). In [4] Kriegl has discussed smooth mappings between locally convex spaces, where a mapping is called smooth iff its composition with smooth curves are smooth. He has compared this concept of smoothness with different $C_{\alpha}^{\infty}$-differentiabilities (see [2]). From [2] and [4] follow that a mapping between Fréchet spaces is smooth iff it is $\mathrm{C}_{c}^{\infty}$-differentiable. Thus the inverse function theorem of Nash and Moser is valid for this concept of smoothness.

\section{REFERENCES}

1. HAMILTON, R.S. The Inverse Function Theorem of Nash and Moser. Bulletin of AMS, New ser. vo1. 7, 65-222 (1982).

2. KELLER, H.H. Differential calculus in locally convex spaces. Lecture Notes in Math. 417, Springer Berlin, 1974.

3. SCHMID, R. The inverse function theorem of Nash and Moser for the $\Gamma$-differentiability. Proc. of the 2nd school on convergence spaces, Schwerin, 1983, Convergence Structures and Applications, II, Berlin 1984.

4. KRIEGL, A. Eine kartesisch abgeschlossene Kategorie glatter Abbildungen zwischen beliebigen lokalkonvexen Vektorräumen. Mh. Math. 95, 287-309 (1983). 


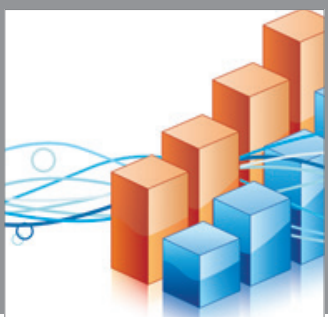

Advances in

Operations Research

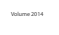

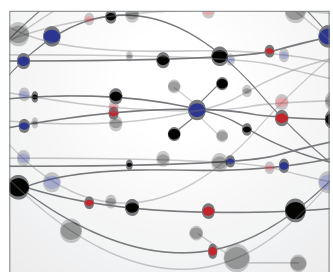

\section{The Scientific} World Journal
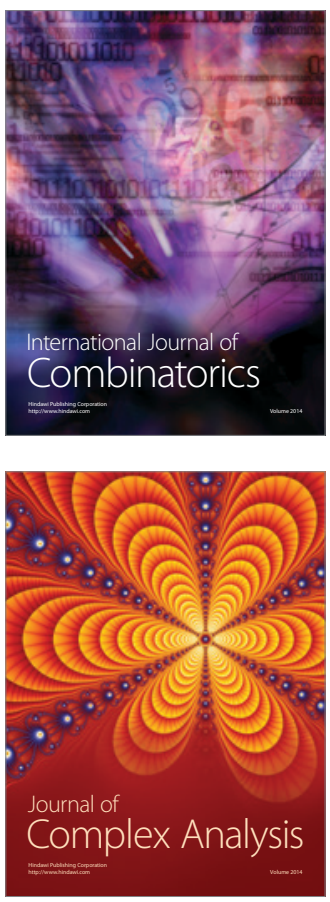

International Journal of

Mathematics and

Mathematical

Sciences
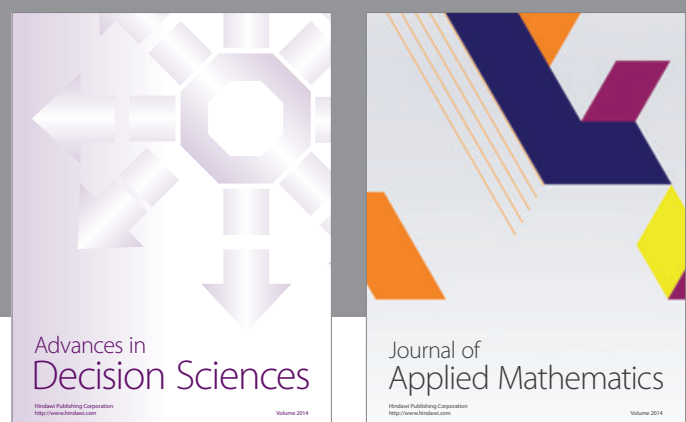

Journal of

Applied Mathematics
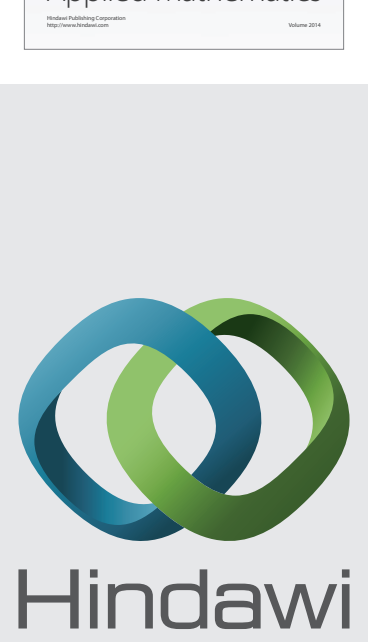

Submit your manuscripts at http://www.hindawi.com
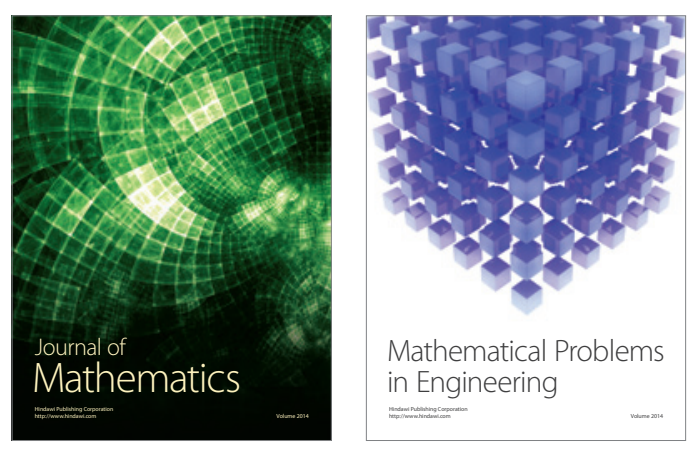

Mathematical Problems in Engineering
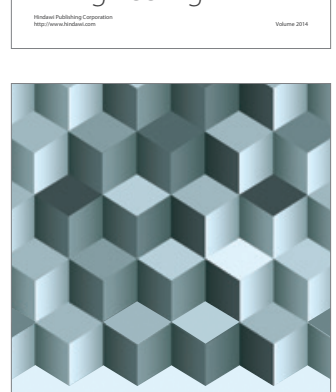

Journal of

Function Spaces
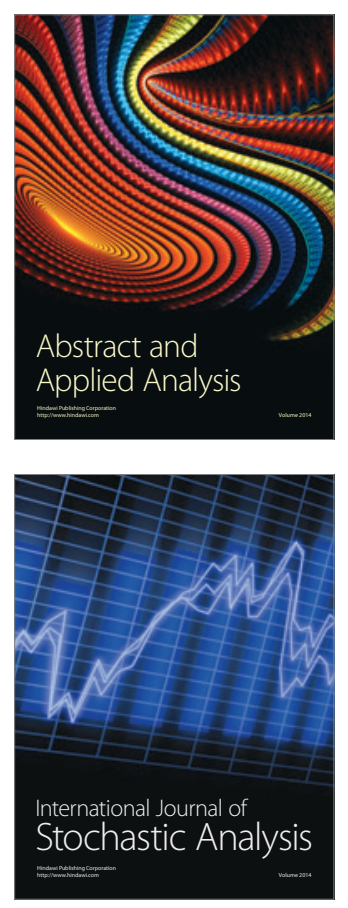

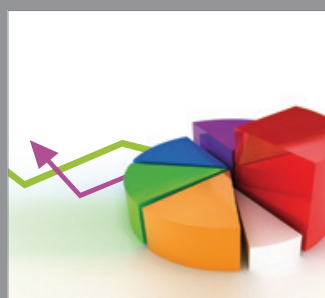

ournal of

Probability and Statistics

Promensencen
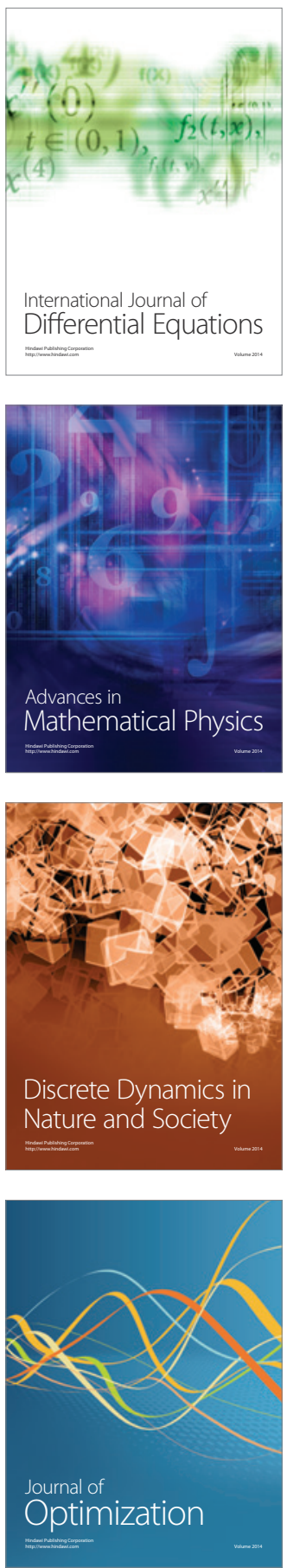